\title{
Effects of Online Social Support and Perceived Social Support on the Relationship Between Perceived Stress and Problematic Smartphone Usage Among Chinese Undergraduates
}

\author{
Chengjia Zhao' \\ Huihui Xu iD \\ Xinyi Lai I \\ Xue Yang ${ }^{2}$ \\ Xiaolian $\mathrm{Tu}^{3}$ \\ Nani Ding ${ }^{4}$ \\ Yijun Lv ${ }^{5}$ \\ Guohua Zhang ${ }^{1,6}$ \\ 'Department of Psychology, School of \\ Mental Health, Wenzhou Medical \\ University, Wenzhou, 325035, People's \\ Republic of China; ${ }^{2}$ Center for Health \\ Behaviours Research, JC School of Public \\ Health and Primary Care, Faculty of \\ Medicine, The Chinese University of \\ Hong Kong, Hong Kong, People's \\ Republic of China; ${ }^{3}$ School of Renji, \\ Wenzhou Medical University, Wenzhou, \\ 325035, China; ${ }^{4}$ School of Public Health \\ and Management, Wenzhou Medical \\ University, Wenzhou, 325035, People's \\ Republic of China; ${ }^{5}$ Wenzhou Medical \\ University, Wenzhou, 325035, People's \\ Republic of China; ${ }^{6}$ The Affiliated \\ Kangning Hospital, Wenzhou Medical \\ University, Wenzhou, 325007, People's \\ Republic of China
}

Purpose: Recent research has identified a relationship between perceived stress and problematic smartphone usage. However, little is known about the underlying mediating and moderating mechanisms. The present study aimed to test the relationship between perceived stress and problematic smartphone usage as well as the mediating role of online social support and the moderating role of perceived social support among Chinese undergraduates. Patients and Methods: A sample of 1123 college students participated in the study. The Perceived Stress Scale, Mobile Phone Addiction Index Scale, Online Social Support Questionnaire, and Perceived Social Support Scale were used.

Results: Perceived stress was significantly and positively associated with problematic smartphone usage, and online social support partially mediated the relationship between perceived stress and problematic smartphone usage. Moderated mediation analysis suggested that perceived social support moderated the association between perceived stress and online social support. This relationship became stronger for college students with lower levels of perceived social support.

Conclusion: The results of this study can contribute to a better understanding of how perceived stress increases the risk of problematic smartphone usage. The importance of reducing online social support and enhancing perceived social support in preventing undergraduates' problematic smartphone usage is highlighted. The findings should be taken with caution given the convenience sampling and cross-sectional study design.

Keywords: problematic smartphone usage, perceived stress, online social support, perceived social support, Chinese undergraduates

\section{Introduction}

Mobile media use has become a necessity of life around the world. Mobile phones expedite communication without restrictions due to physical contiguity or spatial fixation and empower users to perform a variety of online activities, like online e-meeting, online gaming, and other online services. ${ }^{1-3}$ In the past decade, the frequency of smartphone use among young people in Asia and Europe has increased dramatically. ${ }^{4}$ For example, as of July 2020, there were about 932 million smartphone users in China, of which $23.7 \%$ were students. ${ }^{5}$ College students are generally highly motivated to use smartphones and update them quickly, and they are also the fastest adopters and users of new applications and
Correspondence: Guohua Zhang; Yijun Lv Tel +86 0577- 8668 9925; +86 0577-86699III

Email zghcnu@wmu.edu.cn; lyj@wmu.edu.cn 
new programs. ${ }^{6}$ Recent researches show that college students generally own smartphones. ${ }^{7,8}$ What's more, college students are more likely to be addicted to mobile phones, leading to problematic smartphone usage. ${ }^{8}$ For instance, the prevalence of problematic smartphone use of undergraduates was 21.3 to $33.2 \%$ from 2016 to 2019 in China. ${ }^{69}$ Other countries (eg, Romania, Australia, and Eastern Nepal) showed a close approximation of addiction rates from $16 \%$ to $40.9 \%{ }^{10-12}$ This is similar to the approximately $25 \%$ addiction rate found in the systematic review of children and adolescents. ${ }^{13}$

Problematic smartphone use (PSU) refers to smartphone use associated with at least some element of dysfunctional use, such as anxiety when the phone was not available, or neglect of other activities. ${ }^{14,15}$ A systematic review, meta-analysis, and GRADE of the evidence indicate that approximately 1 in 4 children and young people are demonstrating problematic smartphone use, a pattern of behavior that mirrors that of a behavioral addiction. ${ }^{13}$ PSU shares many traits with substance abuse disorders and behavioral addictions, ${ }^{14,15}$ and seems to be widespread. This is not surprising given that those at risk for PSU have similar characteristics to those at risk for other addictions. Just like alcohol, smartphone use is socially accepted and widely used. In addition, smart phones are believed to be beneficial to work, education and leisure. PSU thus poses a different, and arguably bigger, public health problem than substance abuse or even online gaming. ${ }^{13}$ Some researchers have also suggested adding "mobile phone addiction" to the DSM-V as a diagnostic criterion, but it was not accepted because of the lack of empirical research results ${ }^{16}$ and the pathogenesis of mobile phone addiction is poorly understood. ${ }^{17}$ Therefore, the concept of "problematic smartphone use" was adopted in this study.

\section{Perceived Stress and Problematic Smartphone Usage}

Stress is defined as the process in which individuals perceive, assess, and respond to harmful, threatening, or challenging events or stimuli. ${ }^{18}$ Stress has been well documented as a risk element in the development of addiction and the vulnerability to relapse. ${ }^{19}$ From the perspective of general stress theory, ${ }^{20}$ when people experience tension and stress, they will use various coping strategies to relieve tension and eliminate the negative outcomes brought by tension, such as negative life events and life troubles. ${ }^{21}$ Some researchers believe that the main motivation behind problematic behavior is to escape the stress and negative emotions in real life. ${ }^{22}$ Previous studies have also suggested that stress is a trigger for problematic smartphone usage. ${ }^{23,24}$ Furthermore, perceived stress is a better prognosticator of mental health problems than stress, just like depression and physical symptoms, health service use, social anxiety, and reduced maintenance of smoking. ${ }^{25}$ Perceived stress refers to the state or feeling a person has when they feel that they need more than the resources they have to meet that need. ${ }^{25}$ Perceived stress is a critical predictor of problematic smartphone usage in various populations, such as Chinese and Saudi Arabia individuals. ${ }^{23,24}$ Therefore, this study hypothesizes that perceived stress and problematic smartphone usage are positively associated with college students (H1).

\section{The Mediating Role of Online Social Support}

Few studies have tested the underlying processes of the relationship between perceived stress and problematic smartphone usage. Online social support may be a potentially important mediator. Online social support refers to the communication based on virtual space, people are understood and respected in the exchange of emotions, information, and materials, thus gaining an identity and a sense of belonging. ${ }^{26}$ The researchers found that perceived stress was positively correlated with online social support. $^{27,28}$ However, relying heavily on online social support may increase the risk of dependence on the Internet and problematic phone use, leading to Internet addiction $^{29}$ and problematic phone use. ${ }^{30,31}$

According to the compensation model of Internet use, perceived stress and difficulties can motivate people to overindulge in the online world and rely on apps to meet their needs or relieve their irritability. ${ }^{32}$ In turn, excessive use of mobile phones may lead to various addictive behaviors, such as Internet gaming addiction and other addictive behaviors. ${ }^{33}$ Therefore, we believe that perceived stress may prompt individuals to seek online social support, while excessive use of the mobile phone may lead to problematic smartphone usage. A previous study has shown that online social support had a mediating role between stressful events and Internet dependence. ${ }^{27}$ Thus, the study hypothesizes that online social support mediates the relationship between perceived stress and problematic smartphone usage $(\mathrm{H} 2)$. 


\section{The Moderating Role of Perceived Social Support}

Perceived social support refers to the degree to which individuals sense social support from various sources, such as friends, family, and other sources. ${ }^{34}$ Perceived social support contributes to improving their ability to engage their cognitive, emotional, and behavioral responses to stressful events. ${ }^{35,36}$ High perceived social support is associated not only with physical and mental health, but also with psychological resilience, self-esteem, and subjective well-being. ${ }^{37-39}$ Meanwhile, perceived social support can alleviate addictive behaviors, including problematic smartphone usage. ${ }^{31}$

Perceived social support can relieve the impacts of perceived stress on problematic smartphone usage. From the stress buffer hypothesis, positive individual or interpersonal factors can buffer the impact of stress on one's mental and behavioral. ${ }^{40}$ Thus, people who perceived greater social support may have fewer mental or behavioral problems than those with lower perceived social support. Also, empirical studies have revealed that individuals with high perceived social support are well adapted to both low- and high-pressure environments and have a low risk of addiction. ${ }^{41,42}$ Previous studies have proved that perceived social support moderates the influence of perceived stress on subsequent behavior, such as adjustment, ${ }^{42}$ and a sense of integrity, ${ }^{43}$ as well as the impact of stress on negative emotion. ${ }^{44}$ Hence, perceived social support may have a moderating impact on the link between perceived stress and problematic smartphone usage. Besides, college students with low perceived social support may seek more online social support in stressful situations. The Poor-get -Richer model implies that individuals with limited perceived social support may use new communication opportunities to build relationships and obtain support on the Internet. ${ }^{45}$ For instance, socially isolated people are more possible to profit from the Internet if they have a stronger purpose or motivation for online social interaction. ${ }^{46}$ A recent study revealed that undergraduates with low perceptions of social support have a stronger motivation to use networks to relieve pressure in stressful situations, to seek more online social support. ${ }^{47}$ Thus, perceived social support may have a moderating effect on the link between perceived stress and online social support. Furthermore, perceived social support may weaken the negative effects of online social support on problematic smartphone usage. Wu's (2007) research revealed that perceived social support can negatively predict Internet dependence, while online social support can positively predict Internet addiction. ${ }^{48}$ Besides, perceived social support and online social support had obvious interaction effects on Internet addiction as well, the outcomes showed that perceived social support can modulate the relationship between online social support and Internet addiction.

On the strength of the theoretical perspectives and empirical findings, perceived social support may moderate the proposed mediation model among college students. Hence, the present study hypothesizes that the relationships between perceived stress and problematic smartphone usage via online social support are moderated by perceived social support (H3).

\section{The Present Study}

The present study proposes a moderated mediation model to investigate social support matters: Effects of online social support and perceived social support on the relationship between perceived stress and problematic smartphone usage among Chinese undergraduates (Figure 1).

\section{Patients and Methods Participants and Procedure}

This research used convenient sampling to collect data. The participants were recruited at Wenzhou Medical University in Wenzhou City, Zhejiang Province, China. The university has approximately 19,100 students. The inclusion criteria of this study included: 1) willingness to participate in the baseline; and 2) using a smartphone daily. Date from the undergraduate students of eleven majors $(N=1123)$, these included Psychiatry, Clinical medicine, Traditional Chinese medicine, and so on. Data were collected in December 2018. Of the 1150 subjects who completed the survey anonymously, 27 (2.3\%) were excluded from the data analysis because more than $20 \%$ of the data was missing from the scale for one or more key psychological variables. The remaining 1123 (97.7\%) were used for analyses in the following report.

\section{Ethical Statements}

Ethical approval was obtained from the Ethics Committee of Wenzhou Medical University. Participants were fully informed of the investigation before participating in the investigation and had signed the informed consent. In this study, all participants were voluntary, the questionnaire was anonymous, and we also used the non-identification code to ensure the confidentiality of information. This study was conducted following the Declaration of Helsinki. 


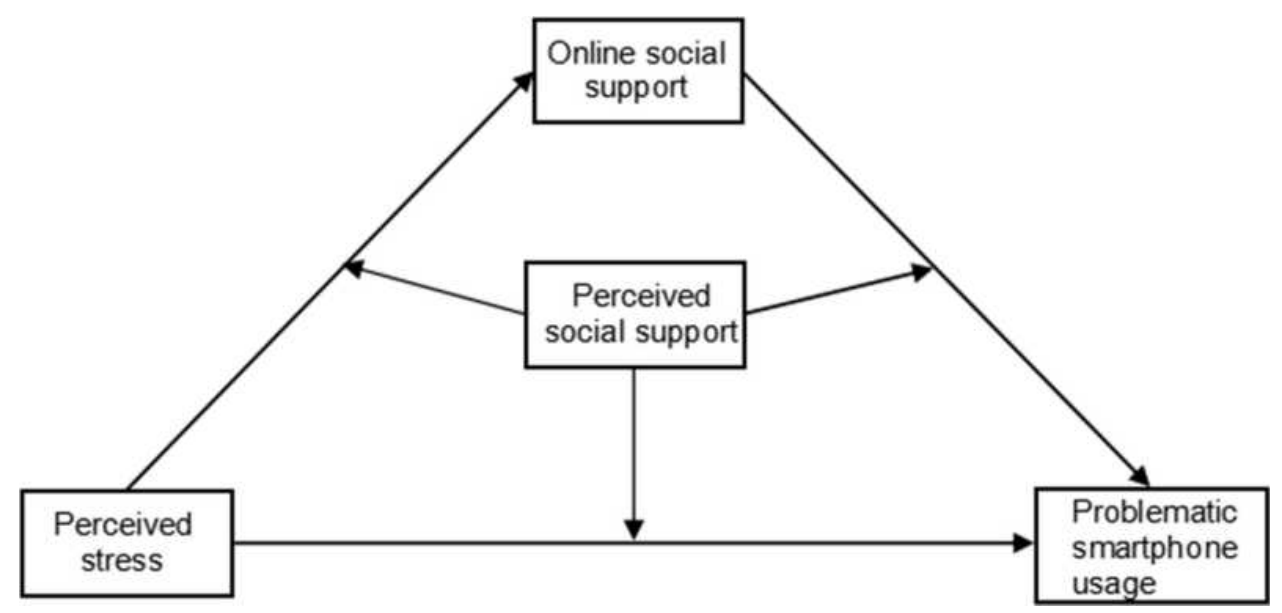

Figure I The proposed moderated mediating model.

\section{Measures}

\section{Perceived Stress Scale}

The 14-item Perceived Stress Scale ${ }^{25,49}$ was used for measuring perceived stress of undergraduates in the past month, an example item is "Feeling upset because something unexpected has happened and losing control of important things in life". Rated on a 5-point response scale $(1=$ not at all, $5=$ very much) higher scores indicate higher levels of perceived stress and a stronger sense of loss of handle. The scale has good reliability and validity and can be used for assessing perceived stress in Chinese populations. ${ }^{49,50}$ Current reliability: the confirmatory factor analysis (CFA) results pointed that the loading range of all factors was $0.57-0.77$, with good fitting: $\chi^{2} / d f=4.57, \mathrm{TLI}=0.94, \mathrm{CFI}=0.96$, RMSEA $=0.057$, SRMR $=0.047$. The Cronbach's $\alpha=0.73$.

\section{Online Social Support Questionnaire}

Online social support was measured by the 23-item Online Social Support Questionnaire. ${ }^{26}$ An example item is "In mobile social media, I can find people to listen to my feelings about personal privacy". Rated on a 5-point response scale ( $1=$ very disagree, $5=$ very agree) with higher scores representing the higher the level of online social support. The scale showed good reliability and validity in Chinese populations. ${ }^{31}$ Current reliability: CFA results showed that the loading range of all factors was $0.59-0.96$, with good fitting: $\chi^{2} / d f=3.61, \mathrm{TLI}=0.94, \mathrm{CFI}=0.95$, $\mathrm{RMSEA}=0.049$ and SRMR $=0.048$. The Cronbach's $\alpha=0.88$.

\section{Mobile Phone Addiction Index Scale}

Problematic smartphone usage was measured by the Mobile Phone Addiction Index Scale, ${ }^{51,52}$ the scale consists of 17 items. In the Mobile Phone Addiction Index Scale, eight items (ie, 3, 5, 6, 7, 8, 10, 15, 16) were revised from Young's eight-item Diagnostic Questionnaire for Internet Addiction ${ }^{53}$ for screening addictive internet use. An example item is "When you're down, you play with your cell phone to improve your mood". Rated on a 5 -point response scale ( $1=$ never, $5=$ always), higher total scores indicate a higher tendency on problematic smartphone usage. The scale showed good reliability and validity in Chinese populations. ${ }^{54,55}$ Current reliability: CFA results showed that the loading range of all factors was $0.53-0.78$, with good fitting: $\chi^{2} / d f=4.28$, $\mathrm{TLI}=0.91, \mathrm{CFI}=0.93, \mathrm{RMSEA}=0.055$ and SRMR $=$ 0.048 . The Cronbach's $\alpha=0.82$.

\section{Perceived Social Support Scale}

Perceived social support was measured by the Perceived Social Support Scale, ${ }^{56,57}$ the scale consists of 12 items. It contains three sources, family (eg, "My family can help me practically and concretely"), friends (eg, "My friends can share happiness and sadness with me"), and others (eg, "I can share happiness and sadness with some people (such as teachers, relatives, classmates)"). Rated on a 7-point response scale ( $1=$ not at all disagree, $7=$ very agree $)$, higher total scores indicate a higher level of perceived social support. This scale showed good reliability and validity in Chinese populations. ${ }^{39,58}$ Current reliability: CFA results showed that the loading range of all factors was $0.47-$ 0.86 , with good fitting: $\chi^{2} / d f=2.24, \mathrm{TLI}=0.99, \mathrm{CFI}=$ 0.99, RMSEA $=0.034$ and SRMR $=0.019$. The Cronbach's $\alpha=0.86$. 


\section{Data Analysis}

CFA was used to test all scales. The chi-square test was used to test the goodness of fit, which included the following indicators, Tucker-Lewis index (TLI), Comparative Fit Index (CFI), Root Mean Square Error of Approximation (RMSEA), and Standard Root Mean-square Residual (SRMR). When the model is fully fitted, $p$-value $>0.05$; While the model fitting is acceptable, the CFI and TLI values $>0.95$, RMSEA and SRMR values are required $>0.08 .{ }^{59}$ Descriptive statistical calculation was performed for independent variables (perceived stress), control variables (sex, family origin), mediator (online social support), moderator (perceived social support), and the outcome variable (problematic smartphone usage). Pearson correlation analysis was used to verify the correlation between these variables. This study did not delete missing values but replaced them with average values. SPSS version 22.0. was used.

The moderated mediation model from Hayes ${ }^{60}$ was used for the following steps. At first, use the PROCESS macro (Model 4) to examine the mediating effect of OSS. Second, we further examined whether PSS on the links between PS and MPA was moderated by PSS, it was analyzed by the PROCESS macro (Model 59). Whether the effects of Model 4 and Model 59 were significant or not was determined by bootstrap confidence interval (CIs) based on 5000 random samples. If 95\% Confidence intervals (CI) are without zero, the influence is considered significant at $\alpha=0.05$.

\section{Results}

\section{Common-Method Bias Test}

In this study, Harman's single factor method was applied to test the common-method bias. ${ }^{61}$ The results suggest that the first common variance factor was $18.06 \%$, which was lower than the critical criterion $(40 \%)$. There was no obvious co-method bias in this study.

\section{Preliminary Analyses}

Data from the participants who completed the survey was reported in this study $(\mathrm{N}=1123), 37.4 \%$ were male. The participants were 346 freshmen (30.8\%), 414 sophomores (36.9\%), and 363 seniors (32.3\%). The background characteristics of the subjects (ie, sex, grade, family origin,) are described in Table 1.

Table 2 shows the means, standard deviations (SDs), and correlations for all the study variables (grade, onechild family, and subject are not significantly related to the outcome variables and are not included in the table). As the results showed, PS was positively correlated with OSS $(r=0.18, p<0.001)$, MPA $(r=0.23, p<0.001)$ and PSS $(r=0.14, p<0.001)$. OSS was positively correlated with MPA $(r=0.13, p<0.001)$ and PSS $(r=0.31, p<0.001)$. The association between MPA and PSS was negative $(r=$ $-.08, p<0.01)$.

\section{Mediation Effect Analysis}

As shown in Table 3, PS was positively associated with MPA $(\beta=0.211, p<0.001)$. Therefore, the results support hypothesis 1 . Our research adopts bias-corrected bootstrapping techniques by Hayes PROCESS macro (Model 4) to test the mediation effect. Controlling for these variables (sex, family origin), the outcomes showed that there was a significant indirect effect via affective commitment with $95 \%$ bias-corrected confidence intervals $[0.003,0.032]$ based on 5000 bootstrapped samples in Table 3 . OSS partially mediated the relationship between

Table I The Participants' Background Characteristics ( $N=\mid$ I23)

\begin{tabular}{|c|c|c|c|c|c|c|c|}
\hline Variables & & $\mathbf{N}$ & (\%) & Variables & & $\mathbf{N}$ & (\%) \\
\hline \multirow[t]{2}{*}{ Sex } & Male & 419 & 37.4 & \multirow[t]{11}{*}{ Subject } & Anesthesia & 51 & 4.5 \\
\hline & Female & 704 & 62.6 & & Forensic & 53 & 4.7 \\
\hline \multirow[t]{3}{*}{ Grade } & Freshman & 346 & 30.8 & & Oral medicine & 57 & 5.1 \\
\hline & Sophomore & $4 \mid 4$ & 36.9 & & Traditional Chinese medicine & 162 & 14.4 \\
\hline & Junior & 363 & 32.3 & & Rehabilitation & 74 & 6.6 \\
\hline \multirow[t]{2}{*}{ Family Origin } & Urban & 636 & 56.6 & & Applied Psychology & 83 & 7.4 \\
\hline & Rural & 487 & 43.4 & & Psychiatry & 85 & 7.6 \\
\hline \multirow[t]{4}{*}{ One-child family } & One-child & 531 & 47.3 & & General medicine & 227 & 20.2 \\
\hline & More than one child & 592 & 52.7 & & Preventive medicine & 90 & 8.0 \\
\hline & & & & & Public management & 121 & 10.8 \\
\hline & & & & & Clinical medicine & 120 & 10.7 \\
\hline
\end{tabular}


Table 2 Descriptive Statistics and Correlations Between Variables

\begin{tabular}{|l|l|l|l|l|l|l|l|}
\hline & Mean & SD & I & $\mathbf{2}$ & $\mathbf{3}$ & $\mathbf{4}$ & $\mathbf{5}$ \\
\hline I.Sex & 0.37 & 0.48 & $\mathrm{I}$ & & & & \\
2.Family Origin & 0.58 & 0.49 & -.004 & 1 & & \\
3.PS & 2.81 & 0.45 & $-.127^{* * *}$ & -.042 & 1 & & \\
4.OSS & 3.30 & 0.64 & -.017 & $-.114^{* * *}$ & $0.177^{* * *}$ & 1 & \\
5.MPA & 2.86 & 0.64 & $-.070^{*}$ & -.038 & $0.23 I^{* * *}$ & $0.125^{* * *}$ & 1 \\
6.PSS & 5.12 & 1.20 & $-.117^{* * *}$ & -.025 & $0.142^{* * *}$ & $0.305^{* * *}$ & $-.077^{* *}$ \\
\hline
\end{tabular}

Notes: $\mathrm{N}=$ I 123. ${ }^{*} \mathrm{p}<0.05,{ }^{*} \mathrm{p}<0.01,{ }^{*} * \mathrm{p}<<0.001$.

Abbreviations: PS, perceived stress; OSS, online social support; MPA, mobile phone addiction; PSS, perceived social support.

Table 3 Bootstrapping Indirect Effects and 95\% Confidence Intervals $(\mathrm{Cl})$ for the Mediation Model

\begin{tabular}{|l|c|c|c|}
\hline $\begin{array}{l}\text { Model } \\
\text { Pathways }\end{array}$ & Effect Size & SE & $\begin{array}{c}\text { Bias-Corrected CI } \\
\text { (95\%) }\end{array}$ \\
\cline { 2 - 2 } & Standarized $\boldsymbol{\beta}$ & & \\
\hline PS $\rightarrow$ MPA & $0.211^{* * *}$ & 0.030 & {$[0.153,0.269]$} \\
PS $\rightarrow$ OSS $\rightarrow$ MPA & $0.014^{*}$ & 0.007 & {$[0.002,0.030]$} \\
Total effect & $0.225^{* * *}$ & 0.029 & {$[0.168,0.283]$} \\
\hline
\end{tabular}

Notes: $\mathrm{N}=1$ 1 23. ${ }^{*} \mathrm{p}<0.05,{ }^{*} *{ }^{*} \mathrm{p}<0.001$.

Abbreviations: PS, perceived stress; OSS, online social support; MPA, mobile phone addiction.

PS and MPA (indirect effect $=0.02, \mathrm{SE}=0.01$ ). The mediation effect accounted for $9.09 \%$ of the total effect of PS on MPA. Therefore, Hypothesis 2 was supported.

\section{Testing for the Moderated Mediation}

Controlling for these variables (sex, family origin), Table 4 shows the results, the product (interaction term) of PS and PSS can significantly predict OSS $(\beta=-.078, p<0.001)$. Based on previous study, ${ }^{19}$ we plotted predicted online social support against PS separately for low and high levels of PSS (Figure 2). Simple slope tests showed that for college students with low PSS, PS significantly predicted OSS $\left(B_{\text {simple }}=0.20, p<0.001\right)$. For individuals with high perceived social support, the predictive effect of PS on OSS became non-significant. Table 4 showed that the product (interaction term) of PS and PSS as well as OS and PSS did not have a significant predictive impact on MPA.

The bias-adjusted percentile bootup analysis further indicated that the indirect effects of PS on MPA were mitigated by PSS through OSS. Specifically, for undergraduates with low PSS, the indirect relationship between PS and MPA was significant $(\beta=0.199, \mathrm{SE}=0.035,95 \% \mathrm{CI}=0.131,0.267)$. For undergraduates with high PSS, the indirect relationship between PS and MPA was not significant $(\beta=0.044, \mathrm{SE}=$ $0.041,95 \% \mathrm{CI}=-.036,0.123)$. In sum, these results indicated that PSS moderated the indirect associations between PS and MPA via OSS. Hence, Hypothesis 3 was partially supported.

\section{Discussion}

In this study, a moderated mediation model has been used to analyze the underlying mechanisms between perceived stress and problematic smartphone usage among Chinese undergraduates. The results showed that the mediating effect of online social support between perceived stress and problematic

Table 4 Testing the Moderated Mediation Effect of Perceived Stress on Mobile Phone Addiction

\begin{tabular}{|l|l|c|c|c|c|}
\hline \multicolumn{1}{|l|}{ Mediated Moderation } & & & \\
\hline 95\%Bias-corrected Confidence Interrals & & & & \\
\hline Dependent variable & Int & Effect & Boot SE & LLCI & ULCl \\
OSS & IntI & $-0.078^{*}$ & 0.024 & -0.126 & -0.030 \\
MPA & IntI & 0.012 & 0.038 & -0.069 & 0.078 \\
MPA & Int2 & -0.006 & 0.038 & -0.090 & 0.060 \\
\hline Dependent variable & Level of PSS & Effect & Boot SE & LLCI & ULCI \\
OSS & Low(-ISD) & $0.199 * * *$ & 0.035 & 0.13 I & 0.267 \\
& Mean & $0.12 I^{* * *}$ & 0.029 & 0.065 & 0.178 \\
& High(+ISD) & 0.044 & 0.041 & -0.036 & 0.123 \\
\hline
\end{tabular}

Notes: $\mathrm{N}=1$ 1 23. ${ }^{*} \mathrm{p}<0.05, * * * \mathrm{p}<0.001$.

Abbreviations: PS, perceived stress; OSS, online social support; MPA, mobile phone addiction; PSS, perceived social support; Int I, PS*PSS; Int2, OSS*PSS. 


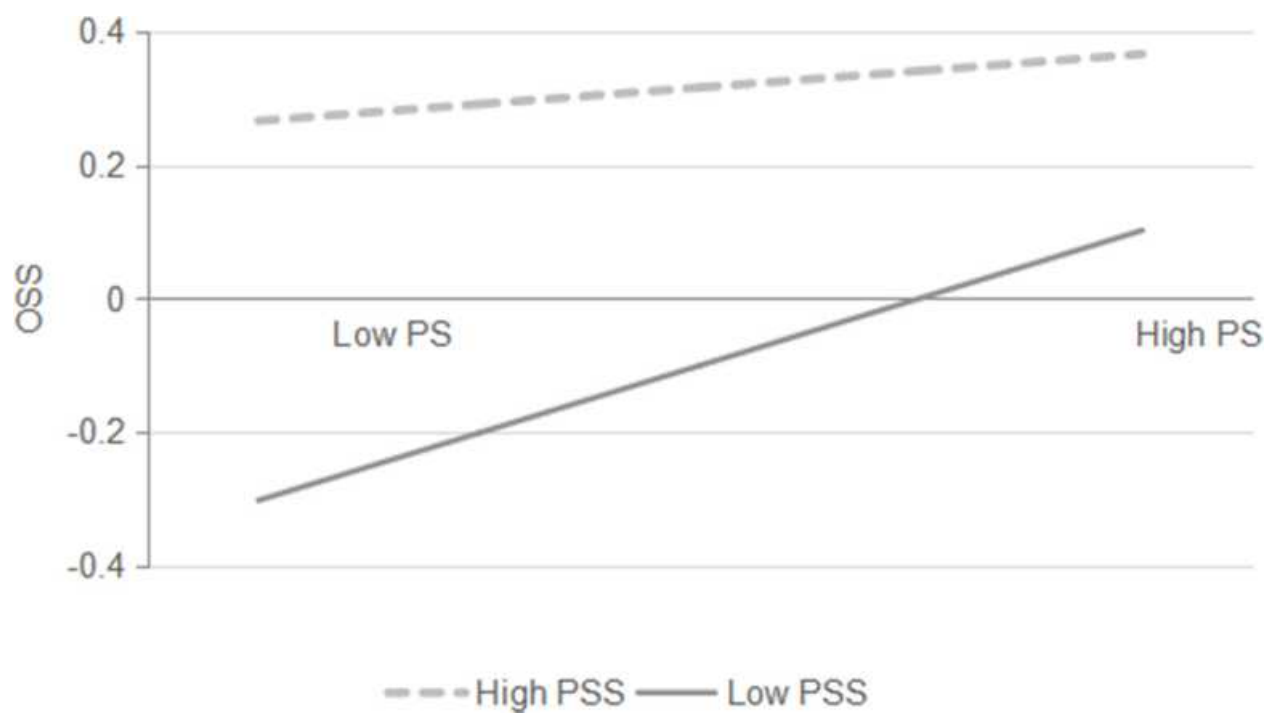

Figure 2 Interaction between PS and PSS on online social support.

Abbreviations: PS, perceived stress; OSS, online social support; PSS, perceived social support.

smartphone usage was moderated by perceived social support. Specifically, the mediating effect of online social support was stronger among college students with lower perceived social support than those with higher perceived social support.

\section{Perceived Stress and Problematic Smartphone Usage}

In this study, perceived stress has a significant positive correlation with problematic smartphone usage; H1 was supported. These results corroborate other cross-sectional studies, ${ }^{23,24}$ and verified the general strain theory. ${ }^{20}$ The previous study has suggested that perceived stress is a hazardous trigger for problematic smartphone usage because it may reduce positive affect and impulse control and increase withdrawal in actual life, leading to passive stress coping, such as spending a great amount of time online to avoid real-life stress. ${ }^{9}$ Studying in universities, especially in the medical education environment is perceived to be stressful, and students may experience stressful events such as psychosocial and academic factors. ${ }^{62}$ In college students, the strong connection between perceived stress and problematic smartphone usage suggests that decreased perceived stress may decrease the risk of problematic smartphone usage. Addiction in this area is not established, but given the frequency of PSU amongst CYP and its significant association with symptoms of common mental disorders, ${ }^{13}$ this relationship and consideration of PSU as a potential causative factor requires urgent further exploration.

\section{The Mediating Role of Online Social Support}

The results of this study indicate that online social support acted as a mediator between perceived stress and problematic smartphone usage; $\mathrm{H} 2$ was supported. This study is the first to prove such a mediation effect. That is, perceived stress can promote the activation of online social support, which in turn intensifies problematic smartphone usage. Therefore, online social support is a result of perceived stress as well as a catalyst for problematic smartphone usage. Also, it's worth regarding that online social support just partially mediated the relationship between perceived stress and problematic smartphone usage. The positive direct relationship between perceived stress and problematic smartphone usage indicates that perceived stress may be the direct factor that significantly increases problematic smartphone usage among undergraduates. Other potential mediators should be explored in future work. For example, self-control and depression/anxiety may mediate the link between perceived stress and problematic smartphone usage. ${ }^{63}$

The results extend the application of the Compensation model of Internet $\mathrm{Use}^{32}$ to explain the relationship between perceived stress and problematic smartphone usage and the role of online social support. This compensation model of Internet use proposes that college students may overuse Internet-connected devices in search of online social support and escape from real-life problems, ${ }^{32}$ which can result in using the Internet excessively and even symptoms of addiction. When college students manage stressors poorly, they 
might seek online social support, ${ }^{28,64}$ in turn increasing the risk of problematic smartphone usage. . $^{27,30,31}$

Given the significant mediation effect of online social support, our study further reveals that individuals who perceived more stress are more possible to seek relief online, and have more online social support, therefore more possible to develops into problematic smartphone usage. The present study indicates that the harmful impact of perceived stress on problematic smartphone usage can be alleviated through intervention to improve online social support. These interventions are warranted for adaptation for use with college students, especially for those who have problems using their smartphones.

\section{The Moderating Role of Perceived Social Support}

H3 was partially supported. First, we found that perceived social support regulates the link between perceived stress and online social support. The impact of perceived pressure on online social support among undergraduates with low perceived social support was stronger than the influence on people with a high sense of social support. The result is consistent with the poor-get-richer model, ${ }^{45}$ indicating that college students with limited perceived social support are quite possible to use new communication opportunities to build relationships and obtain supportive relationships in stressful situations. With the increase in stress, college students with low perceived social support have a stronger motivation to relieve stress by using the Internet as a distraction and seek more social support online. ${ }^{47}$

Unexpectedly, we find no moderating effect of perceived social support on the relationship between online social support and problematic smartphone usage. This is inconsistent with the previous research results. ${ }^{48}$ One plausible argument is that we use overall social support (the total score for perceived social support), not support from specific sources, such as family, friends, and others. For example, there is evidence that offline perceived social support has a significant main effect on online access support and college students' Internet addiction tendency, but the interaction was not significant. However, subjective social support and emotional support not only had significant main effects on Internet addiction tendency but also had a significant negative interaction. ${ }^{65}$

Surprisingly, perceived social support has not moderated the link between perceived stress and problematic smartphone usage, which is inconsistent with our hypothesis. The outcome may not be verified for the stress buffer hypothesis, ${ }^{40}$ which suggests that perceived stress is a significant hazardous trigger for problematic smartphone usage among Chinese college students, and perceived social support does not buffer the negative effect of perceived stress on problematic smartphone usage. We explain this result in two ways. First of all, we replace relationship-specific sources of support with comprehensive social support. The ability of perceived social support to relieve stress may vary from source to source. ${ }^{16}$ For instance, findings showed that support from family can alleviate the negative effect of stress on college students' Internet addiction, while support from friends does not, ${ }^{66}$ at the same time, a study has found support from family and friends can provide college students with protective functions against stress. ${ }^{67}$ Second, there may be situational and/or crosscultural differences. Jin et al (2014) reported that support from either the work or family domain moderated the relations between role stress and work-family conflict in an American sample but not in a Chinese sample. ${ }^{68}$ Our findings suggest that relationship-specific sources and situational and/or cultural differences in support should be considered when examining the moderating effect of perceived social support on the link between perceived stress and problematic smartphone usage among undergraduates.

\section{Limitations}

Several limitations should be noted. Firstly, we used convenient samples of college students, which will cause sampling bias, and thus the generalization of these findings should be taken seriously. Future studies could extend the model to diverse populations. Besides, the research depended on a self-report survey. Future research could use a variety of information providers (eg, peers and parents) and research methods (eg, qualitative interviews) to collect data to better understand individuals' mental and behavioral status. Second, the cross-sectional design prevents the testing of causal hypotheses. It is possible that the relationships among the studied variables are reciprocal. For example, a longitudinal study has shown a reciprocal relationship between perceived stress and problematic smartphone usage. $^{43}$ Thus, future work should examine the mediation model with a longitudinal study design. Third, As the subjects in this study are from a university, it may not be conducive to the promotion of our research results. Future studies may explore the model in different populations. Fourth, we only looked at the moderating effect of overall social support. Future research could explore the moderating effects of support of family, friends, and other types of support separately. Such as parental support can modulate 
the association between stress and Internet addiction, but friend support does not. ${ }^{66}$ Moreover, friend support and family support have different moderating effects for community violence and post-traumatic stress disorder. ${ }^{69}$ Fifth, this study failed to identify groups according to SAR (Specific Absorption Rate) values of smartphones, it has been found that radiofrequency (RF) and microwave (MW) can have some effects in biological organisms, ${ }^{70}$ and individuals exposed to RF and MW have different physical and mental health compared to the normal group. ${ }^{71,72}$ Future research could classify groups according to SAR values of smartphones. After classifying they can observe a relation between problematic smartphone usage and SAR values.

\section{Conclusion}

In summary, this research is among the first to explore the mediating impact of online social support and the moderating role of perceived social support underlying the link between perceived stress and problematic smartphone usage among Chinese undergraduates. This research discovered that online social support played a mediating role between perceived stress and problematic smartphone usage, while perceived social support played a moderating role in the indirect effect of perceived stress on problematic smartphone usage. The effect of perceived stress on problematic smartphone usage via online social support was stronger for college students with lower perceived social support than for those with higher perceived social support. The results of this study lead to a better understanding of how perceived stress increases the risk of problematic smartphone usage and the importance of appropriately reducing the use of virtual networks for stress relief and social support from the Internet among undergraduates. The findings should be taken with caution given the convenience sampling and cross-sectional study design.

\section{Date}

The datasets in the study are available from the corresponding author on reasonable request.

\section{Acknowledgments}

All authors have made substantial contributions to the research design, data collection, analysis, and interpretation; participated in drafting and critically revising articles; agreed to submit to the current journal; approved the version to be published; and agreed to be responsible for all aspects of the research.

\section{Funding}

This research was funded by the Youth Project of the National Social Science Foundation of China [Grant No. CBA170257].

\section{Disclosure}

The authors declare no conflicts of interest in this work.

\section{References}

1. Parnes P. Secure Inclusion of Phones into Online E-Meetings. Berlin: Heidelberg; 2003.

2. Botta F, Moat HS, Preis T. Quantifying crowd size with mobile Phone and Twitter data. R Soc Open Sci. 2015;2(5):150162.

3. Hong W, Liu R-D, Ding Y, Zhen R, Jiang R, Fu X. Autonomy need dissatisfaction in daily life and problematic mobile phone use: the mediating roles of boredom proneness and mobile phone gaming. Int J Environ Res Public Health. 2020;17(15):5305.

4. Billieux J, Maurage P, Lopez-Fernandez O, Kuss DJ, Griffiths MD. Can disordered mobile phone use be considered a behavioral addiction? An update on current evidence and a comprehensive model for future research. Current Addiction Reports. 2015;2(2):156-162.

5. CNNIC. The 46th China Statistical Report on Internet Development. 2020.

6. Qudah MFA, Albursan IS, Bakhiet SFA, et al. Smartphone addiction and its relationship with cyberbullying among university students. Int J Ment Health Addict. 2019;17(3):628-643.

7. Soo-Koung J. The emotional factors influencing mobile phone dependency of college students and gender difference. Aquatic Marine Educ. 2019;31(4):1193-1200.

8. Zhang G, Yang X, Tu X, Ding N, Lau JT. Prospective relationships between mobile phone dependence and mental health status among Chinese undergraduate students with college adjustment as a mediator. J Affect Disord. 2020;260:498-505.

9. Chen B, Liu F, Ding S, Ying X, Wang L, Wen Y. Gender differences in factors associated with smartphone addiction: a cross-sectional study among medical college students. BMC Psychiatry. 2017;17 (1):341.

10. Gligor Ș, Mozoș I. Indicators of smartphone addiction and stress score in university students. Wien Klin Wochenschr. 2019;131 (5-6):120-125. doi:10.1007/s00508-018-1373-5

11. Oviedo-Trespalacios O, Nandavar S, Newton JDA, Demant D, Phillips JG. Problematic use of mobile phones in australia ...Is it getting worse? Front Psychiatry. 2019;10:15.

12. Thapa K, Lama S, Pokharel R, Sigdel R, Rimal SP. Mobile phone dependence among Undergraduate Students of a Medical College of Eastern Nepal: a descriptive cross-sectional study. J Nepal Med Assoc. 2020;58(224):234-239.

13. Sohn SY, Rees P, Wildridge B, Kalk NJ, Carter B. Prevalence of problematic smartphone usage and associated mental health outcomes amongst children and young people: a systematic review, meta-analysis and GRADE of the evidence. BMC Psychiatry. 2019;19(1):356.

14. Bianchi APJ. Psychological predictors of problem mobile phone use. CyberPsychol Behav. 2005;8(1):39-51.

15. Kwon M, Lee J-Y, Won W-Y, et al. Development and validation of a Smartphone Addiction Scale (SAS). PLoS One. 2013;8(2):e56936.

16. Lee C-YS, Goldstein SE. Loneliness, Stress, and Social Support in Young Adulthood: does the Source of Support Matter? $J$ Youth Adolesc. 2016;45(3):568-580.

17. Bian M, Leung L. Linking loneliness, shyness, smartphone addiction symptoms, and patterns of smartphone use to social capital. Soc Sci Comput Rev. 2015;33(1):61-79. 
18. Sinha R. Chronic stress, drug use, and vulnerability to addiction. Ann N Y Acad Sci. 2008;1141:105-130.

19. Wei. Influence of Stress on Internet Addiction and Its Mechanism. Wuhan: Central China Normal University; 2014.

20. Agnew RJC. Foundation for a general strain theory of crime and delinquency. Criminology. 1992;30(1):47-88.

21. Jun S, Choi E. Academic stress and Internet addiction from general strain theory framework. Comput Human Behav. 2015;49:282-287.

22. Stetina BU, Kothgassner OD, Lehenbauer M, Kryspinexner I. Beyond the fascination of online-games: probing addictive behavior and depression in the world of online-gaming. Comput Human Behav. 2011;27(1):473-479.

23. Long J, Liu TQ, Liao YH, et al. Prevalence and correlates of problematic smartphone use in a large random sample of Chinese undergraduates. BMC Psychiatry. 2016;16(1):408.

24. Venkatesh E, Jemal MYA, Samani ASA. Smart phone usage and addiction among dental students in Saudi Arabia: a cross sectional study. Int J Adolesc Med Health. 2017;31:1.

25. Cohen S, Kamarck T, Mermelstein R. A global measure of perceived stress. J Health Soc Behav. 1983;24(4):385-396.

26. Liang X, Wei L. A preliminary study on the evaluation of online social support for College Students. Psychol Sci. 2008;3:671-689.

27. Xiao. Research on the Current Situation of College Students' Internet Addiction Tendency and Its Relationship with Life Events and Online Social Support. Changsha: Hunan Normal University; 2010.

28. Dam AEH, de Vugt ME, van Boxtel MPJ, Verhey FRJ. Effectiveness of an online social support intervention for caregivers of people with dementia: the study protocol of a randomised controlled trial. Trials. 2017;18(1):395.

29. Liu C, Ma J-L. Adult attachment orientations and social networking site addiction: the mediating effects of online social support and the fear of missing out. Front Psychol. 2019;10.

30. Han. Research on the Relationship and Intervention Between Mobile Phone Dependence and Self-Esteem and Social Support Among College Students. Zhangzhou: Zhangzhou normal university; 2012.

31. Jiang Y, Bai X. On the relationship between college students' mobile phone addiction and justice: the responsibility role of online social support. Special Educ China. 2014;(1):6.

32. Kardefeltwinther D. Problematizing excessive online gaming and its psychological predictors. Comput Human Behav. 2014;31 (31):118-122

33. Subrahmanyam K, Šmahel D. When is It Too Much? Excessive Internet Use and Addictive Behavior. In: Digital Youth: The Role of Media in Development. New York, NY; 2011:157-178.

34. Wang X, Wang X, Ma H. Rating Scales for Mental Health (In Chinese). Beijing: Chinese Mental Health Journal Press; 2009.

35. Cohen S. Social relationships and health. Am Psychol. 2004;59 (8):676-684.

36. Klink JL, Byars-Winston A, Bakken LL. Coping efficacy and perceived family support: potential factors for reducing stress in premedical students. Med Educ. 2008;42(6):572-579.

37. Itzick M, Kagan M, Talkatz P. Perceived social support as a moderator between perceived discrimination and subjective well-being among people with physical disabilities in Israel. Disability Rehabilitation. 2018;40(18):2208-2216.

38. Banstola RS, Ogino T, Inoue S. Self-esteem, perceived social support, social capital, and risk-behavior among urban high school adolescents in Nepal. SSM - Population Health. 2020;11:100570.

39. Huang Y, Wu R, Wu J, Yang Q, Zheng S, Wu K. Psychological resilience, self-acceptance, perceived social support and their associations with mental health of incarcerated offenders in China. Asian J Psychiatr. 2020;52:102166.

40. Cohen S, Wills TA. Stress, social support, and the buffering hypothesis. Psychol Bull. 1985;98(2):310-357.
41. Cole DA, Nick EA, Zelkowitz RL, Roeder K, Spinelli T. Online social support for young people: does it recapitulate in-person social support; can it help? Comput Human Behav. 2017;68:456-464.

42. Vats N, Kaur N. Perceived social support and perceived stress, relations to adjustment among migrant students: a test of buffering hypothesis. Indian J Public Health Res Develop. 2018;9 (12):1780-1787.

43. Liu J, Wang Q. Influence of daily stress on migrant Children's sense of integrity: the moderating effect of Social support and adversity belief. Psychol Develop Educ. 2018;34(5):548-557.

44. Hou L, Zhang Z. Stress and anxiety symptoms of medical graduate students: mediating effect of negative emotion and regulating effect of social support. Chinese $J$ Behav Med Brain Sci. 2019;12:1108-1112.

45. Valkenburg PM, Schouten AP, Peter J. Adolescents' identity experiments on the internet. New Media Soc. 2005;7(3):383-402.

46. Bessiere K, Kiesler S, Kraut RE, Boneva B. Effects of internet use and social resources on changes in depression. Inf Commun Soc. 2008;11(1):47-70.

47. Chu X, Huang W, Li Y, Lei L. Perception of stress and the use of problematic online games for college students: a mediated model. J Clin Psychol. 2020;28(2):379-383.

48. Wu J. Influence of social support on Internet addiction. Inf Soc Res. 2007; 7:173-189.

49. Hou X-L, Wang H-Z, Guo C, Gaskin J, Rost DH, Wang J-L. Psychological resilience can help combat the effect of stress on problematic social networking site usage. Pers Individ Dif. 2017;109:61-66.

50. Wang J, Wang H, Gaskin J, Wang L. The role of stress and motivation in problematic smartphone use among college students. Comput Human Behav. 2015;53:181-188.

51. Leung L. Linking psychological attributes to addiction and improper use of the mobile phone among addiction in Hong Kong. $J$ Child Media. 2008;2(2):93-113.

52. Huang $\mathrm{H}$, Niu L, Zhou C, Wu H. Reliability and validity test of the Chinese version of mobile phone dependence index in college students. Chinese J Clin Psychol. 2014;22(5):835-838.

53. Young KS. Internet addiction: the emergence of a new clinical disorder. Cyberpsychol Behav. 1998;1(3):237-244.

54. Gao Q, Sun R, Fu E, Jia G, Xiang Y. Parent-child relationship and smartphone use disorder among Chinese adolescents: the mediating role of quality of life and the moderating role of educational level. Addict Behav. 2020;101:106065.

55. Huang Q, Li Y, Huang S, et al. Smartphone use and sleep quality in Chinese College Students: a preliminary study. Front Psychiatry. 2020;11:352.

56. Zimet GD, Dahlem NW, Zimet SG, Farley GK. The multidimensional scale of perceived social support. J Pers Assess. 1988;52 (1):30-41.

57. Yan BZ. Research on the relationship between social support, self-esteem and subjective well-being of college students. Psychol Develop Educ. 2006;3:60-64.

58. Miao L, Feng J, Wu L, Zhang S, Ge Z, Pan Y. The mediating role of general self-efficacy in the association between perceived social support and oral health-related quality of life after initial periodontal therapy. BMC Oral Health. 2016;16(1):68.

59. Hu L, Bentler PM. Cutoff criteria for fit indexes in covariance structure analysis: conventional criteria versus new alternatives. Struct Equation Modeling. 1999;6(1):1-55.

60. Hayes AF. Introduction to Mediation, Moderation and Conditional Process Analysis: A Regression-Based Approach. Guilford publications; 2017.

61. Hair J. Multivariate Data Analysis. 2009. 
62. Borjalilu S, Mohammadi A, Mojtahedzadeh R. Sources and severity of perceived stress among Iranian Medical Students. Iran Red Crescent Med J. 2015;17(10): 17767.

63. Elhai JD, Dvorak RD, Levine JC, Hall BJ. Problematic smartphone use: a conceptual overview and systematic review of relations with anxiety and depression psychopathology. $J$ Affect Disord. 2017;207:251-259.

64. Ding D, Xiao N, Zhang, X. Relationship between life events, online social support and Internet addiction tendency. J Educ Sci Hunan Normal Univ. 2013;12(6):118-121.

65. Kong Y. Research on the Relationship Between Social Support, Loneliness and Internet Addiction Tendency of College Students. Qufu: Qufu Normal University; 2010.

66. Wei H, Zhou Z, Zhang Y, Ding Q. Relationship between stress and Internet addiction: the regulatory role of family support and friend support. Psychol Behav Res. 2018;16(2):266-271.

67. Bland HW, Melton BF, Welle P, Bigham L. Stress tolerance: new challenges for millennial college students. Coll Stud J. 2012;46 (2):362-376
68. Jin J, Xu S, Wang Y. Comparison of Role stress, work-family conflict and psychological Depression between China and the United States the moderating effect of social support. Acta Psychologica Sinica. 2014;46(8):1144-1160.

69. Affrunti NW, Suárez L, Simpson D. Community violence and posttraumatic stress disorder symptoms in urban youth: the moderating influence of friend and parent support. J Community Psychol. 2018;46(5):636-650

70. Dasdag S, Balci K, Celik M, et al. Neurologic and biochemical findings and $\mathrm{CD} 4 / \mathrm{CD} 8$ ratio in people occupationally exposed to RF and microwave. Biotechnol BiotechnolEquipment. 1992;6 (4):37-39.

71. Oto R, Akdağ Z, Daşdağ S, Celik Y. Evaluation of psychologic parameters in people occupationally exposed to radiofrequencies and microwave. Biotechnol BiotechnolEquipment. 1994;8(4):71-74.

72. Meric F, Dasdag S, Dasdag MM. Does radiofrequency exposure affect hearing of children? J Int Advan Otol. 2009;5:3.
Psychology Research and Behavior Management

\section{Publish your work in this journal}

Psychology Research and Behavior Management is an international, peer-reviewed, open access journal focusing on the science of psychology and its application in behavior management to develop improved outcomes in the clinical, educational, sports and business arenas. Specific topics covered in the journal include: Neuroscience, memory and decision making; Behavior modification and management; Clinical

\section{Dovepress}

applications; Business and sports performance management; Social and developmental studies; Animal studies. The manuscript management system is completely online and includes a very quick and fair peer-review system, which is all easy to use. Visit http://www. dovepress.com/testimonials.php to read real quotes from published authors. 